\title{
Un sistema de acceso a la educación superior técnico-profesional: ideas a partir del caso de Chile
}

\author{
Roberto Flores, ${ }^{a}$ Catalina Iglesias, ${ }^{a}$ Ricardo D. Paredes ${ }^{b}$ \\ a Centro de Estudios Duoc UC, Chile \\ b Escuela de Ingeniería, Pontificia Universidad Católica de Chile, Chile
}

\begin{abstract}
RESUMEN: En este artículo describimos los principales desafíos que conlleva implementar un sistema de admisión en el subsector técnico-profesional (TP) de educación superior. Si bien la tarea es progresivamente necesaria para un conjunto de países, tiene carácter de urgente en el caso de Chile, donde la nueva Ley 21.091, promulgada en 2018, estableció como obligatoria tal implementación para un conjunto significativo de instituciones. La ausencia de tradición,
\end{abstract}

\footnotetext{
Roberto Flores es ingeniero civil industrial y Magíster en Ciencias de la Ingeniería, Pontificia Universidad Católica de Chile. Actualmente se desempeña como investigador senior del Centro de Estudios Duoc UC, Santiago, Chile.

Catalina Iglesias es ingeniera civil industrial, Pontificia Universidad Católica de Chile, y Máster en Administración Pública, Universidad de Columbia, EEUU. Actualmente se desempeña como subdirectora del Centro de Estudios Duoc UC, Santiago, Chile.

Ricardo D. Paredes es ingeniero comercial, Universidad de Chile, y PhD, Universidad de California, EEUU. Es profesor de la Escuela de Ingeniería, Pontificia Universidad Católica de Chile. Dirección: Av. Vicuña Mackenna 4860, Macul, Santiago, Chile, CP 7810000. Email: rparedes@ing.puc.cl.

Este artículo se benefició de la discusión interna en Duoc UC, así como de los comentarios de los miembros del grupo de discusión de políticas de acceso CRUCH-TP, de los miembros del Comité de Acceso de la Educación Técnico-Profesional, de tres árbitros anónimos de Estudios Públicos y, en particular, de los aportes de Daniela Torre y Marcela Arellano.
} 
la gran oferta de programas y la experiencia de un sistema descentralizado y poco selectivo - aun cuando se le reconocen méritosson obstáculos objetivos que se suman a aprensiones de parte de instituciones que podrían beneficiarse de un sistema no coordinado. La propuesta de implementación de este sistema en el subsector $\mathrm{TP}$, válida para un amplio espectro de instituciones, incluyendo universidades no selectivas, debiera considerar una transición. Esta podría minimizar el costo de errores de diseño del sistema, el que sugerimos debiera ser: i) centralizado, lo que reduce los costos de postulación; y ii) flexible, lo que permitiría el uso de diversos instrumentos afines con los objetivos particulares de las instituciones. Proponemos un sistema aplicable en dos fases: en primera instancia, una postulación centralizada a través de una plataforma única y con matrícula posterior en el programa asignado según las vacantes; la segunda, a realizarse al acabar la primera, similar al proceso vigente que prevalece en la mayor parte de los subsectores técnico-profesionales en América Latina, que es sin postulación y con matrícula directa.

PALABRAS ClaVE: sistema de acceso, centralización, selectividad, subsector técnico-profesional, Ley de Educación Superior

RECIBIDO: octubre 2019 / ACEPTADO: marzo 2020

\section{An access system to higher vocational-technical education: Ideas based on the Chilean experience}

ABSTRACT: In this article, we describe the main challenges involved in implementing an admission system in the technical-professional (TP) subsector of higher education. Although the task is progressively necessary for a group of countries, it is urgent in the case of Chile, where the new Law 21,091, enacted in 2018, established such implementation as mandatory for a significant group of institutions. The lack of tradition, the large number of programs on offer and the experience of a decentralized and unselective system - even when it is recognized for its merits - are objective obstacles that add to the apprehensions of institutions that could benefit from an uncoordinated system. The proposal to implement this system in the TP subsector, valid for a wide range of institutions, including non-selective universities, should consider a transition. This could minimize the cost of system design errors, which we suggest should be: (i) centralized, which reduces application costs; and (ii) flexible, which would allow the use of various instruments related to the particular objectives of the institutions. We propose a system applicable in two phases: first, a centralized application through a single platform and with subsequent enrollment in the assigned program according to vacancies; a second alternative is without application and with direct enrollment — an alternative to be implemented after the first 
phase, similar to the current process prevailing in most of the technical-professional subsectors in Latin America.

KEYwORDS: access system, centralization, selectivity, vocational technical sub-sector, Law of Higher Education

RECEIVED: October 2019 / ACCEPTED: March 2020

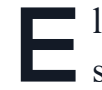

sistema tradicional universitario de admisión en la educación superior en Chile es centralizado. Esto conlleva un conjunto de instrumentos comunes para las instituciones que lo componen, las que se atienen a procedimientos conjuntos de postulación (fechas, normas de aceptación, entre otros). Este sistema fue exclusivo del grupo de las universidades pertenecientes al Consejo de Rectores de Universidades Chilenas (CRUCH), organismo que agrupa a las instituciones universitarias estatales y privadas creadas antes de 1981. El sistema, fuertemente subsidiado por el Estado, ha sido cuestionado debido a que ha sido muy reticente a incorporar a nuevas instituciones y la adscripción ha dependido de la voluntad de los incumbentes, lo cual ha llegado a constituir una virtual 'barrera de entrada'. Aunque el CRUCH limitó durante un tiempo el ingreso de otras universidades a su sistema de admisión, desde el proceso de admisión 2011 ha incorporado a 13 universidades. Así, desde 2013 el sistema de acceso a la educación superior pasó a denominarse Sistema Único de Admisión (SUA).

Al año 2020 aún existe una porción considerable del subsistema universitario, así como la totalidad del sistema técnico-profesional (TP) - compuesto por Centros de Formación Técnica (CFT) e Institutos Profesionales (IP)—, que no forman parte del SUA y que manejan políticas particulares de postulación y matrícula. Con la entrada en vigencia de la nueva Ley de Educación Superior, en mayo de 2018 (Ley 21.091), se estableció, entre otras materias, la creación de dos sistemas de acceso, uno para las universidades y otro para el sector TP, obligatorios para todas las instituciones que adscriban al beneficio de gratuidad para sus estudiantes. Específicamente, la ley establece que "el Sistema deberá contemplar procesos e instrumentos de acceso de aplicación general para las instituciones adscritas, que considerarán las particularidades de cada subsistema". Así también, que los "instrumentos (...) que puedan establecer las instituciones de educación superior deberán respetar los principios que rigen al Sistema de Acceso". De lo anterior se desprende 
que cada subsistema debe establecer procesos e instrumentos para la postulación y admisión, los que deben ser objetivos y transparentes y, asimismo, deben considerar, entre otros, la diversidad de capacidades, trayectorias y talentos previos de los estudiantes.

Entre los aspectos más relevantes del sistema de acceso, que deberá entrar en funcionamiento en 2020 para el proceso de admisión 2021, la norma señala que: i) operará a través de una plataforma electrónica única — administrada por la Subsecretaría de Educación Superior- que dispondrá de información actualizada asociada a la oferta académica, procesos de admisión, mecanismos e instrumentos de selección; ii) existirán dos comités técnicos (universitario y técnico-profesional) integrados por los rectores respectivos de distintos tipos de instituciones de educación superior (IES) y por el subsecretario de Educación Superior; iii) cada comité definirá los procesos e instrumentos de aplicación general, sin perjuicio de instrumentos propios de las IES, que cuenten con autorización de los comités; y iv) los requisitos de admisión y selección en cada carrera o programa los determinarán las instituciones.

Por último, y muy importante respecto del diseño del sistema, cabe remarcar que la Ley 21.091 establece en el articulado transitorio que, cuando entre en vigencia el sistema de acceso, la restricción individual que rige el crecimiento de la matrícula impuesta a cada institución adscrita a gratuidad, dejará de operar. Ello, aunque no está explícito, sugiere que la restricción individual pasará a reemplazarse por una total. Esto último, siendo más eficiente, solo es posible de realizar cuando existe un sistema de admisión que entrega un orden para el conjunto de los postulantes del sistema.

El propósito de este artículo es describir los desafíos que conlleva la implementación de un sistema de acceso en el sector TP, en un contexto en el que ya opera uno para el sector universitario, así como proponer caminos para su implementación. El sector TP no solo carece de instrumentos y procedimientos generales de selección, sino que históricamente no ha sido selectivo; no obstante lo cual, a partir de una evaluación general, muestra que el sistema ha operado razonablemente.

El artículo se estructura, a continuación, en tres secciones. La siguiente contextualiza la discusión y describe el sistema de admisión a la educación superior que opera en Chile. Luego se identifican los elemen- 
tos centrales del debate relativos al sector TP. Por último se concluye con una propuesta específica de implementación que, si bien es particular al caso chileno, aplica perfectamente a otras realidades.

\section{Problemas abordados por los sistemas de admisión}

Un análisis adecuado de los sistemas de admisión requiere distinguir entre dos conceptos que suelen ser incorrectamente mezclados en la discusión: centralización y selectividad. Centralización remite a la integración o coordinación de distintas instituciones y sus mecanismos de acceso. Un sistema centralizado define criterios de aceptación comunes, plazos de implementación simultáneos y eventualmente usa plataformas integradas. Selectividad remite a las exigencias contenidas en los criterios utilizados por parte de las instituciones para escoger entre los postulantes; esos criterios pueden ser comunes a todo el sistema, en cuyo caso el sistema es o no selectivo, o pueden estar asociados a instituciones específicas.

Así, un sistema de admisión puede ser descentralizado y cada institución puede ser selectiva o no serlo. Tal es el caso, por ejemplo, del subsistema brasilero universitario. En este, por mucho tiempo cada universidad aplicó distintas pruebas a sus respectivos postulantes y tuvo diferentes criterios de admisión (muy descentralizado), y coexistieron instituciones muy selectivas con otras que no lo eran. Otro ejemplo es Canadá, donde el sector TP cuenta con un sistema descentralizado estatalmente, pero centralizado a nivel de cada estado. Dependiendo del exceso de demanda, las instituciones canadienses tienen distinto grado de selectividad, para lo cual utilizan la preparación previa del postulante como criterio. En el caso de Chile, el sistema de admisión escolar público (municipal y privado subvencionado) fue completamente descentralizado hasta el año 2019, coexistiendo escuelas de muy distinto grado de selectividad. El diagnóstico arrojó que ese sistema llevaba a una excesiva selección por parte de algunas instituciones y se condujo a cambiarlo hacia uno completamente centralizado, con el uso de un criterio que impedía la selección relacionada con los ingresos de las familias.

Por otro lado, existen diversas experiencias acerca del grado de coordinación entre los subsectores TP y universitario. Mientras en países como Finlandia, Francia, Alemania y Reino Unido el proceso de admisión se desarrolla en conjunto con el universitario, otros casos como Australia consideran postulaciones diferenciadas (Mineduc 2019). 


\section{Centralización}

La centralización en un sistema de selección tiene como propósito optimizar la asignación de recursos entre las instituciones. Si cada institución tiene su propio sistema, asociado a altos costos fijos (como plataforma, elaboración de pruebas de selección y otros), y si la postulación repetida es costosa, un único sistema ahorra recursos tanto para las IES como para los estudiantes. Más relevante aún es que la menor transparencia en la información asociada a un sistema no coordinado, reduce la coincidencia entre las preferencias de los postulantes y las vacantes totales. Por ello, la postulación descentralizada podría generar efectos adversos en la equidad, especialmente porque quienes asumen mayores costos de repetir exámenes son personas de menor ingreso (Espinoza et al. 2017).

En la misma dirección, un sistema descentralizado establece mayores restricciones a un control eficiente de vacantes por parte de la autoridad central. El reciente caso de Chile es ilustrativo en este sentido. Con la introducción de la política de gratuidad, aplicable a un subconjunto de instituciones, se previó un aumento en la matrícula de estas. Para contener el costo y mientras no entraran en vigencia los respectivos sistemas de acceso, el crecimiento de la matrícula en dichas instituciones quedaría limitado a un 2,7\% anual. Ahora bien, la restricción aplicada a nivel institucional lleva a que en el límite no haya una distribución óptima de vacantes. Así, por ejemplo, podrían coexistir zonas con presencia de instituciones sin cupos, pese a una demanda latente, con otras con exceso de vacantes en zonas que carecen de esa demanda, y todo producto de la restricción global del 2,7\% mencionado. Esto sugiere que el término de la restricción individual establecida en la Ley de Educación Superior, cuando entre en vigencia el nuevo sistema de acceso, obedece a la intención del legislador de sustituirla por restricciones de crecimiento globales o, al menos, articuladas. Y ello solo es posible a través de un sistema de acceso centralizado.

Por último, la postulación centralizada permite evitar el problema conocido de unraveling. Este se refiere a la contratación excesivamente temprana, antes de que toda la información relevante se encuentre disponible para los postulantes, para un determinado trabajo (Halaburda 2009). La ausencia de un período específico de postulación y ma- 
trícula incentiva a las instituciones a progresivamente ir adelantando dichos procesos y a extenderlos por un mayor plazo con la finalidad de obtener ventajas respecto de su competencia. Niederle et al. (2009) sostiene que dicho fenómeno puede ser la causa de fallas de mercado, especialmente cuando la información crítica no está disponible en los tiempos en que los individuos toman decisiones. ${ }^{1}$

Roth $(1984,1991)$ analiza los problemas de alineación entre expectativas o intereses de instituciones y estudiantes, situaciones que pueden producirse debido a la falta de información precisamente motivada por el unraveling. Su caso de estudios se desarrolla en el ámbito de la educación, particularmente en la selección de internos de medicina por parte de instituciones de salud estadounidenses y británicas, y en la adecuación a los intereses de estos que buscan continuar sus estudios de especialidad. A su vez, desarrolla cómo dichos problemas pueden ser solucionados a través de un sistema centralizado de postulación, lo que corrige el costo de la postulación y la información dispersa. La elección, el costo y la adecuación de preferencias pueden ser sustancialmente diferentes cuando existe información clara que facilita comparaciones. En esta línea, Abdulkadiroglu et al. (2017) identifican que una oferta de educación no coordinada genera una alineación poco óptima entre los intereses de las instituciones y de los estudiantes. Mientras, Ekmekci y Yenmez (2014) abordan los incentivos necesarios para promover la participación de la totalidad de las escuelas públicas en el sistema centralizado de cuatro ciudades estadounidenses. Sostienen, además, que instituciones con propios regímenes de admisión poseen ventajas producto de la posibilidad de ofertar en períodos de tiempo diferentes, pero que generan el consecuente problema de unraveling.

Una experiencia reciente de transición hacia un sistema de admisión centralizado en educación superior universitaria y TP, es el caso de Brasil. Al respecto, Machado y Szerman (2016) reportan impactos

\footnotetext{
${ }^{1}$ En Chile, la validez hoy en día de este argumento, a diferencia del período en el que se formalizó el subsistema de educación superior técnico-profesional, a principios de la década de 1980, radicaría fundamentalmente en el crecimiento de instituciones y programas, lo que hace sustancialmente más compleja la elección y comparación.
} 
positivos de este cambio en el perfil de los estudiantes finalmente matriculados en las instituciones, así como en la movilidad geográfica.

Evidentemente, un sistema centralizado posee ciertos riesgos, siendo los más relevantes el exceso de burocratización en la admisión y la falta de flexibilidad. Este último aspecto es importante tenerlo en cuenta, dado que la alta flexibilidad es un atributo relevante en el caso chileno del sistema TP.

\section{Selectividad}

El segundo elemento que define un sistema de admisión es la selectividad, es decir, la inducción para elegir a postulantes con determinadas características, por sobre otros que no las tienen. Ya sea por la conveniencia de seleccionar a quienes tienen mayores probabilidades de 'hacerlo mejor', de desarrollar mayor potencial, por un criterio de justicia social o, simplemente, por la necesidad de asignar cupos a una demanda excesiva, en un sistema de admisión se suelen aplicar diferentes criterios para seleccionar. Esos criterios pueden ser más o menos inclusivos y, dependiendo de los casos específicos y de los proyectos educativos de cada institución, se escogerán instrumentos diferentes de selección por institución.

Una buena síntesis de los criterios que se pueden usar para evaluar los sistemas de admisión la plantea Aequalis (2019). Este propone como principios la equidad en el acceso, la integralidad en la respuesta (además del foco en el acceso, debe existir una preocupación por variables futuras como permanencia o desempeño), la articulación entre niveles formativos, y la pertinencia de los instrumentos de evaluación y caracterización. En una línea similar, Orr et al. (2017) sugieren centrarse en tres aspectos: i) equidad que introduce al sistema —quién accede a la educación superior-; ii) efectividad, relacionada con la retención, desempeño y titulación oportuna; y iii) eficiencia en la alineación entre preferencias de los postulantes y vacantes por carrera. Aunque todos los criterios mencionados podrían conllevar cierta priorización, los de equidad e integralidad de la respuesta (o efectividad) se asocian más directamente con la selectividad. Así, por ejemplo, pruebas relacionadas con el conocimiento como criterio de selección, aplicadas en sociedades en las cuales la calidad de la educación es muy desigual, pueden ser especialmente selectivas. 
Tabla 1. Criterios de admisión en países seleccionados

\begin{tabular}{ll}
\hline Pais & \multicolumn{1}{c}{ Funcionamiento } \\
\hline Canadá & $\begin{array}{l}\text { La definición de instrumentos es descentralizada a nivel provincia. } \\
\text { Se consideran las calificaciones de la enseñanza media. El número } \\
\text { de vacantes y el área de interés de la institución pueden jugar un rol } \\
\text { mayor que el de las calificaciones previas. }\end{array}$ \\
Holanda & $\begin{array}{l}\text { Educación superior técnico-profesional se dicta mayormente en uni- } \\
\text { versidades. Los instrumentos de admisión son definidos por autorida- } \\
\text { des centrales, sin perjuicio de que las instituciones pueden incorporar } \\
\text { ponderaciones propias. No existen requisitos restrictivos de entrada. }\end{array}$ \\
Portugal & $\begin{array}{l}\text { Se requiere diploma de educación media y rendición de prueba de } \\
\text { acceso. Prueba única para las instituciones estatales. Instituciones } \\
\text { privadas poseen sus propias pruebas. } \\
\text { Criterios de postulación definidos a nivel central, pero cada institu- } \\
\text { ción puede incorporar variables de selección autónomamente. } \\
\text { Las instituciones poseen libertad para definir los instrumentos de } \\
\text { admisión. Se requiere certificado de educación media, exámenes } \\
\text { de ingreso, experiencia laboral o habilidades en matemáticas y len- } \\
\text { guaje. } \\
\text { Variables de entrada definidas a nivel central, entre las que se en- } \\
\text { cuentran licencia de enseñanza media, experiencia laboral y posi- } \\
\text { ción de la institución dentro de las preferencias de los estudiantes. } \\
\text { Las instituciones pueden complementar con pruebas particulares o } \\
\text { entrevistas personales. } \\
\text { Las instituciones definen sus propios instrumentos, en un marco } \\
\text { definido a nivel central. }\end{array}$ \\
\hline
\end{tabular}

Fuente: UDP-Vertebral (2019).

Una vez definidos y consensuados los principios o criterios del sistema de acceso, el paso siguiente natural es hallar los instrumentos y mecanismos que permitan alcanzarlos. Al respecto, el dilema central tiene relación con que la elección de instrumentos que favorecen un objetivo determinado, suelen desfavorecer otro. Por ejemplo, elegir según la vulnerabilidad favorece la equidad, pero podría también perjudicar la efectividad académica. También se podría optar por elegir privilegiando el rendimiento futuro del estudiante, omitiendo el hecho de que solo utilizando este criterio se podría estar excluyendo a grupos más desaventajados. Sin embargo, es posible encontrar instrumentos que favorezcan un objetivo sin ir en desmedro de otro. Por ejemplo, Gil, Paredes y Sánchez (2013) muestran que la inclusión del Ranking de Notas 
de Enseñanza Media, bonificación de puntaje en función de la posición relativa de un estudiante con respecto a los resultados históricos de su colegio, puede aumentar la equidad sin reducir la efectividad en el caso del subsector universitario.

La Tabla 1 resume distintos instrumentos de admisión a nivel comparado. En ella puede observarse que, sin perjuicio de la libertad de ponderar y a veces elegir instrumentos, existen exigencias a nivel central.

\section{Sistema de admisión universitaria en Chile}

Dada su larga tradición y carácter centralizado en un porcentaje importante de instituciones, cualquier propuesta de acceso al sector TP que considere algún grado de centralización, debe observar el desarrollo y situación presente de la admisión universitaria. A mediados del siglo XIX ya es posible distinguir herramientas para admitir a alumnos en la universidad usadas transversalmente (Bernasconi y Rojas 2003). El mayor número de instituciones y el aumento de la demanda, avanzado el siglo XX, requirió jerarquizar de alguna forma a los postulantes en base a sus resultados, también para favorecer el éxito académico de los mismos estudiantes. Ello dio pie a la creación de la Prueba de Aptitud Académica (PAA), la que fue utilizada masivamente a partir de 1967. Años más tarde se incorporó un nuevo instrumento complementario a la PAA, las notas de enseñanza media (NEM). Con estos dos mecanismos, las instituciones establecían distintos requisitos de ingreso, dependiendo del perfil o características de cada carrera (DEMRE 2010, 2016).

En 2003, la PAA dio paso a la Prueba de Selección Universitaria (PSU), un instrumento de evaluación que utiliza como base los contenidos mínimos obligatorios del plan de formación general de la enseñanza media. Una de las últimas modificaciones de instrumentos fue la incorporación del ranking de notas de enseñanza media en el proceso de admisión 2013, que se instrumentalizó como una bonificación a la NEM, en caso de que el estudiante hubiese obtenido una nota superior al promedio histórico de su colegio.

Hasta el año 2011, las universidades del CRUCH eran las únicas partícipes del sistema de admisión, y sus pruebas y plataformas de asignación de estudiantes fueron financiadas por el Estado. Ello no impidió 
que otras universidades utilizaran algunos de los instrumentos en sus propios procesos, pero la asignación de alumnos a base de las preferencias no les estaba disponible. A partir de 2011 se abrió parcialmente el sistema de admisión a otras universidades privadas, las que previa autorización del Consejo de Rectores pueden participar del proceso integrado de admisión. Al año 2019, de un total de 60 universidades con registro de matrícula, 41 participan de él.

Así, al año 2020 el sistema de acceso centralizado en Chile considera un conjunto de instrumentos, incluyendo la PSU, las NEM y el ranking. Cada universidad perteneciente al sistema comunica las vacantes que tiene para cada una de sus carreras y las ponderaciones que hará de los mencionados instrumentos, para así jerarquizar a los postulantes.

Por su parte, los estudiantes se informan de las ponderaciones que se les exigen en cada institución y carrera, y en la medida en que hayan rendido las pruebas que la postulación requiere pueden postular a hasta un máximo de diez carreras. Cerrado el proceso de postulación, se aplica un algoritmo que asigna a los postulantes según el orden de sus preferencias; ello, en estricto orden de puntaje ponderado según lo establecido por cada institución y según las vacantes.

Cada universidad y cada carrera pueden ponderar los distintos puntajes de la manera que deseen, debiendo considerar necesariamente los resultados de los tres indicadores mencionados. De esa forma, cada estudiante tiene un puntaje diferente dependiendo de la carrera a la cual postule.

Para la asignación opera una regla en la que si el alumno queda en su primera preferencia (por ejemplo, hubo vacantes suficientes como para que el último seleccionado en esa preferencia tenga su mismo o menor puntaje), el sistema acepta esta postulación, eliminando las otras de menor preferencia. Si no hubiera cupos para su primera preferencia, entonces busca para su segunda y así sucesivamente. En caso de no encontrar cupos en todas sus preferencias, el alumno no puede matricularse en ninguna carrera mediante admisión ordinaria en las universidades adscritas al sistema.

Una vez comunicados los resultados de las postulaciones, se abre un proceso de matrícula que, consistente con las asignaciones presupuestarias estatales, se efectúa de manera anual, debiendo atenerse las instituciones miembros a las fechas establecidas para cada etapa (DEMRE 2019). 
De la descripción del proceso se puede colegir que el sistema centralizado no es en sí mismo selectivo, sino que las instituciones, dependiendo de las ponderaciones de cada instrumento, pueden llegar a serlo. Por ejemplo, en la medida en que la PSU se relaciona con el ingreso de la familia, entonces una universidad con alta demanda y que pondere fuertemente a este instrumento será muy selectiva en términos del estatus económico. Por su parte, si una universidad con alta demanda pondera solo el ranking de notas, que en todo tipo de liceos y colegios, independientemente del ingreso de los padres, premiará a los alumnos de mejores notas, si bien dejará a muchos postulantes fuera, será menos selectiva en una métrica de riqueza familiar.

En síntesis, un sistema centralizado en particular es selectivo solo si obliga a cada institución a ponderar un instrumento selectivo con un mínimo relativamente alto. En el caso de Chile, el SUA obliga a cada institución participante a ponderar distintos instrumentos en un porcentaje mínimo.

\section{Acceso en el caso TP}

La educación técnico-profesional en Chile se inicia en la Colonia, cuando diversas órdenes religiosas la imparten como una forma de llegar fundamentalmente a niños necesitados. A nivel superior, la semilla se siembra en la primera mitad del siglo XX con la fundación de la Universidad Técnica Federico Santa María (1931) y de la Universidad Técnica del Estado (1947). Siguió a ello la introducción de carreras técnicas en varias universidades, pero el desarrollo diferenciado, de un sector TP a nivel superior, tiene en Chile una historia relativamente reciente. En 1981, el DFL1 del Ministerio de Educación (Mineduc) dio la posibilidad de crear Centros de Formación Técnica e Institutos Profesionales. Estos fueron inicialmente asociados a la capacitación para la empresa, lo cual, sumado a la fuerte escasez de recursos públicos de esos años, explica que esas instituciones no recibieran financiamiento estatal, se les permitiera el lucro y tuvieran una proliferación inicial que no puso cuidado en la calidad de las instituciones creadas (Paredes y Sevilla 2015; Paredes 2018). Así, con financiamiento eminentemente privado y con el valor de sus aranceles como factor que racionaba los excesos de demanda, no se vio para aquellas en los orígenes la necesidad de contar con un sistema de admisión. 
Desde el año 2006, con la entrada en vigencia del Crédito con Aval del Estado, junto con el proceso de consolidación del sector superior TP y la alta cobertura de la enseñanza media, la matrícula se expandió a niveles no vistos en el subsector. La mayor demanda fue satisfecha con más instituciones y con la ampliación de sus vacantes, lo que permitió, en pocos años, llegar a una cobertura en educación superior similar al promedio de los países de la OCDE (Comisión de Financiamiento 2012). ${ }^{2}$ En este proceso, las IES TP generaron individualmente procesos de matrícula donde, como regla general, se llenaban las vacantes por orden de postulación.

Sin perjuicio de que las IES TP han usado sus propios mecanismos de admisión, todas ellas están afectadas por los plazos que establece el Ministerio de Educación para que los estudiantes accedan a los beneficios financieros que entrega el Estado y que, sin excepción, son determinantes clave de sus matrículas (ver Anexo). Además, existen instancias de coordinación entre instituciones TP, las que han tenido distintos grados de interlocución con las autoridades gubernamentales (Consejo Asesor de Formación TP, red de CFT estatales, Vertebral, entre otras). Por su parte, la Estrategia Nacional de Formación Técnico-Profesional publicada en 2018, cuya principal función es orientar el desarrollo e implementación de las políticas públicas en esta materia (Ley N² 21.091), sostiene que las condiciones de acceso deben ser definidas por cada institución, pero que el sistema de admisión debieran compartirlo las instituciones TP.

Como se ha sostenido, a nivel institucional el sistema TP no está coordinado ni de manera interna ni con el universitario. La relación entre ambos sistemas se da por diversos medios, pero, para los efectos de admisión, aquella por la vía de la sustitución que pueden visualizar los estudiantes, es la más evidente. Así, y pese a que existe mucha variedad en el tipo y calidad entre las instituciones, Ruiz-Tagle y Paredes (2019), en un trabajo de elección de institución, observan que si bien hay un grupo importante de universidades que dominan las preferencias de los estudiantes, existe también una alta sustitución entre un amplio grupo

\footnotetext{
${ }^{2}$ En 1990, a casi diez años desde su creación, había 81 IP y 161 CFT. El proceso se consolidó luego. Hacia 2006, su número se había reducido a 45 IP y 76 CFT, y en 2018, a 43 y 47 , respectivamente.
} 
de universidades menos selectivas y al menos una institución TP de alta calidad.

A la evidencia señalada se suma aquella referida a la sensibilidad en la matrícula de un tipo de institución cuando se afecta la matrícula de otro tipo. La Figura 1 muestra que el hito 'resultado de la PSU', que permite a la mayoría de los estudiantes saber con relativa precisión las posibilidades que tienen de ser aceptados en las universidades de mayor reputación, coincide fuertemente con saltos en la matrícula en Duoc UC, una institución que captura alrededor del $20 \%$ de la matrícula del sector TP.

Figura 1. Postulación a Duoc UC en el período de admisión 2018 (total 82.309)

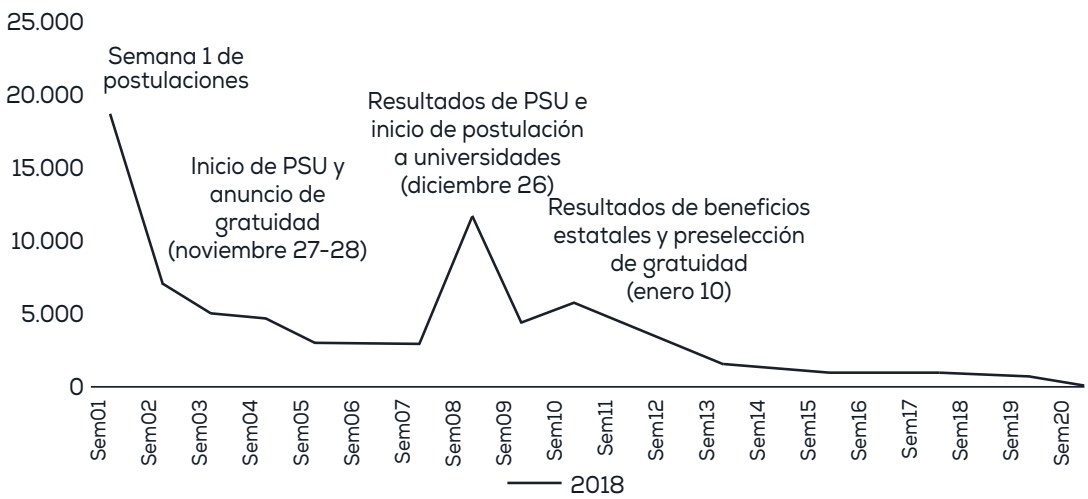

Fuente: elaboración propia en base a datos institucionales de Duoc UC.

La idea de que la educación superior puede realizarse en distintas instituciones, cuyo grado de sustitución es alta, llama a integrar, para evitar el problema de unraveling y los otros costos señalados. A su vez, aquella relación de sustitución entre distintos tipos de IES llama a evaluar el sentido que tiene contar con dos subsistemas. Habiendo sustitución entre instituciones de distinto tipo, como lo son universidades e IES TP, es esperable que aquella sea sustancialmente mayor cuando se trata de instituciones del mismo tipo.

Mientras mayor sea la sustitución desde la perspectiva de los estudiantes, menos probable es que alguna institución por si sola inicie un 
proceso de acceso virtuoso, fijando un período inmutable, compatible con, por ejemplo, el término de los ciclos en educación media. ${ }^{3}$ Cualquier período fijado por un actor induce a sus 'competidores' a adelantar el propio. En otros términos, existiría un efecto 'fuga de postulantes' para dichas instituciones. La evidencia del progresivo adelantamiento de los procesos de admisión en el sector TP, apoya esta hipótesis.

La educación superior TP y también aquella universitaria más masiva de pregrado que no está en el SUA, no explicita instrumentos para el acceso, aunque los tiene. Implícitamente acepta que el resultado académico de los estudiantes no se asocia o no vale la pena asociarlo con ninguna variable objetivable. Así, en el subsector TP, la generalidad de los procesos de admisión y selección consideran una inscripción y matrícula por orden de postulación. Ahora bien, existen algunas excepciones como Duoc UC, que desde el año 2016 decidió priorizar en base a un polinomio que considera NEM, ranking y proveniencia de la educación media TP. También es excepción el CFT estatal de Los Lagos, donde se priorizan alumnos con reconocimiento de aprendizajes previos. En la misma línea, diversos estudios y documentos estratégicos han planteado, por ejemplo, la importancia de profundizar en la articulación entre los niveles formativos (Aequalis 2019; Mineduc 2018; Sevilla et al. 2014).

\section{Un sistema de acceso para el subsector TP}

\section{¿Por qué un sistema?}

Cabe preguntarse por qué se requiere un sistema de acceso para el sector TP cuando el sistema vigente ha funcionado. Responder esto es fundamental, pues ni el debate previo a la Ley de Educación Superior - relativamente menor-, ni 'la historia de la Ley' permiten hacerlo, y ello permitiría dirimir la discusión respecto de qué tipo de sistema exige la norma.

La respuesta que sugerimos es que el legislador visualizó que el creciente financiamiento estudiantil otorgado por el Estado, reforzado en un proceso que llevaría a una virtual gratuidad universal, implica-

\footnotetext{
${ }^{3} \mathrm{El}$ adelantamiento del proceso de matrícula ha hecho que los alumnos que terminan su ciclo escolar no alcancen a tener la licenciatura de educación media al momento de realizar su postulación.
} 
ría en algún momento una presión sobre la matrícula que haría imposible financiarla con los escasos fondos públicos, a menos que se hiciera a costa de la calidad. Esta presunción adquiere especial sentido cuando se considera que con la gratuidad introducida en el año 2016, por glosa presupuestaria, se limitó el crecimiento de la matrícula al 2,7\% anual a nivel institucional. Más relevante aún es que luego la ley estableció que, una vez que estuviera en operación el sistema de acceso, la restricción del 2,7\% dejaría de tener efecto, de lo que se desprende que dicha restricción debiera transitar a una articulada a nivel sistema.

Entonces, lo anterior supone y requiere de un sistema de admisión centralizado, objetivo y transparente. Entendemos que un sistema de acceso en el subsector TP debe ir más allá de la entrega de información a los postulantes, cosa que por lo demás no habría requerido de una ley. Este nuevo sistema centralizado corregiría las ineficiencias a las que nos hemos referido y daría la posibilidad de incorporar ciertos instrumentos de selección o priorización a las instituciones.

A partir de la última premisa señalada, la primera discusión que se debe enfrentar es respecto de los criterios e instrumentos que deben emplearse para dicha priorización. Para acotarla debe reconocerse que mientras por historia y objetivos declarados se suele argumentar que el sector tiene acceso universal, ello no es así. Si bien los aranceles en el sector han sido relativamente menores al del sector universitario, ellos han sido una fuente de restricción al acceso.

Siguiendo a Orr et al. (2017) y atendiendo los objetivos para el sector explicitados en la propia ley, deben reconocerse los trade-offs entre distintos propósitos que pueden asignársele a un sistema de admisión. En lo esencial, la política pública debe equilibrar entre un objetivo amplio de inclusión, referido a la equidad en el acceso de personas jóvenes y adultas, con la efectividad en la formación, lo cual se traduce en priorizar a quienes tengan mayor vocación, facilidad, posibilidad de articulación y, por cierto, se registre un egreso oportuno. Este dilema ha estado muy presente también en el caso de la selección universitaria, donde el instrumento PSU fue complementado con el ranking de notas, opción que no ha estado exenta de polémicas (Brunner 2012). 


\section{Los desafíos de un nuevo sistema en un modelo sin tradición}

Introducir un sistema de acceso centralizado en un sector sin tradición es complejo. Espinoza et al. (2017) advierten sobre la resistencia que ello provocaría especialmente por parte de instituciones de menor calidad, que precisamente se benefician de la confusión de sistemas descentralizados y matriculan a alumnos que de otra forma, más informada, no avanzarían en dicho proceso. Sin perjuicio de lo anterior, cabe reiterar que en Chile existen exigencias para todas las IES, incluyendo las TP en cuanto a proveer información ordenada y cumplir con determinados plazos para que sus alumnos postulen a beneficios estudiantiles de parte del Estado.

No obstante, el mayor costo potencial de pasar a un sistema centralizado es la pérdida de flexibilidad. En el sistema vigente, los estudiantes se matriculan presencialmente en las mismas sedes, sin que medie un proceso de postulación. En esa instancia se produce un proceso iterativo, en el cual las instituciones tienen flexibilidad para manejar el exceso o falta de postulantes en sus programas, lo que permite adaptar las preferencias del posible alumnado con las vacantes disponibles. De este modo, postulantes sin posibilidad de matrícula debido a sobredemanda, pueden ser guiados a tomar carreras similares (o la misma en otra sede o jornada) que sí cuentan con vacantes disponibles. Con esto, y si el proceso de orientación establecido por las instituciones es realizado virtuosamente, sin incurrir en la inducción incorrecta de los estudiantes, son beneficiados los alumnos y las mismas IES.

La necesidad de una iteración se entiende incluso más cuando se verifica el alto número de programas y nomenclaturas a las que se ha llegado en Chile, así como la existencia de matrículas varios días después del inicio del semestre. Esto, más que nada, sugiere un abuso de la flexibilidad mencionada. Una idea de la dimensión del problema y confusión se adquiere cuando se considera que en la oferta total de carreras 2018, las universidades y las ITP sumaron 4.208 y 6.280 programas, respectivamente. Más aún, a diferencia del subsector universitario, en el TP las instituciones con mayor matrícula ofertan una misma carrera en distintas sedes o jornadas.

En definitiva, los riesgos de pasar del régimen completamente descoordinado — como es el actual — a uno centralizado — como el aplicado en el sistema universitario-, son altos. Si pensamos que el alumno 
debe priorizar entre un número acotado de alternativas, la desinformación respecto de sus verdaderas opciones les puede llevar a descartar de su consideración algunas que sí, las cuales, tras una guía adecuada, podrían ser vistas como opciones posibles. Este problema se acota, pero no se elimina, cuando a los alumnos se les ofrecen más posibilidades de escoger (por ejemplo, más de las diez que contempla el sistema de acceso universitario), ya que el desconocimiento entre los postulantes, al menos en la primera etapa, es muy alto.

\section{Una propuesta para el caso de Chile}

La propuesta que sigue, si bien está específicamente focalizada para Chile, considera factores generales como constituyen los riesgos asociados al descalce entre oferta de vacantes y preferencias, lo que ocurriría de replicar un sistema centralizado como el universitario vigente. Al respecto, dos consideraciones son el pilar de la propuesta. En primer lugar, no es deseable sobresimplificar el sistema, dejándolo únicamente como una plataforma de información. Esta no solo ya existe, no requiere de una ley y, como lo señalamos, va en contra del sentido de la misma, sino que mantendría una brecha clara respecto del sistema universitario. Dicha sobresimplificación postergaría la resolución de los problemas que ya se han detallado y que conllevan un sistema descentralizado, particularmente el de unraveling y el de sustituir la restricción de crecimiento a la matrícula que rige hasta la entrada en vigencia del nuevo sistema de acceso. La propuesta de sistema de acceso centralizado a la educación superior debe conciliar la necesidad de una transición, junto con abordar los problemas centrales que tiene la descentralización.

En segundo lugar, es clave cuidar la flexibilidad, que reconoce que las instituciones individualmente, por las razones específicas de sus proyectos educativos, han ido desarrollando sistemas propios que tienen particulares virtudes. Así, la predominancia de algún objetivo como equidad o efectividad debiera ser, con los resguardos que imponga el Estado como responsable de asignar los recursos que finalmente llegan a los estudiantes, de libre determinación por parte de las IES. De hecho, las disposiciones de la ley dan cierta holgura a definiciones propias del Sistema de Acceso, quedando estas bajo la responsabilidad de distintas entidades como los comités de acceso o el Mineduc. En definitiva, las 
instituciones TP que adscriban al sistema debieran tener libertad de optar a los instrumentos (que ha de aprobar el comité del subsector TP), entendiendo que ellos deben tener relación con los objetivos educacionales que persigue la ley y que además deben ser conocidos y verificables por la instancia que defina el proceso de selección. ${ }^{4}$

El sistema que proponemos, que aplica tanto para carreras técnicas como profesionales del mundo TP, consta de dos fases o etapas. La primera, bastante similar a la que en la actualidad ejecuta el SUA, a diferencia de aquella considera un conjunto de criterios no contemplados en la admisión universitaria, como pudieran ser la procedencia de la educación media, el avance a partir del reconocimiento de aprendizajes previos, nuevas pruebas validadas que se relacionen con la vocación y otros.

En la primera fase, habiendo definido los instrumentos, ya sea como requisito de postulación o como elemento para priorización, las instituciones comunicarán a la Subsecretaría de Educación Superior las ponderaciones que ocuparán para cada uno, y las vacantes por carrera, sede y jornada que dispondrán.

La Subsecretaría difundirá los criterios, informará las vacantes, en conjunto con los indicadores de calidad de cada una de las instituciones, incluyendo empleabilidad, salarios, acreditación por carreras y otras variables pertinentes, a través de la plataforma dispuesta por la Subsecretaría para efectos de la postulación.

Los estudiantes debieran postular ordenadamente según sus preferencias durante un período definido por la Subsecretaría, a un conjunto de máximo 20 opciones, siempre a través de una plataforma. Aunque no es imperativo que el proceso universitario y TP coincidan por completo, sí debiera existir cierta coordinación de fechas y plazos, en particular durante esta primera etapa y, evidentemente, entre las partes del subsistema universitario. ${ }^{5}$

Como resultado de este proceso, y una vez cerrado el plazo de postulación, el sistema entregará a los estudiantes el aviso de dónde han

\footnotetext{
${ }^{4}$ El uso del ranking de notas por parte de Duoc UC fue un factor importante de la propuesta conceptual del sistema de admisión aplicado en la institución a partir de 2016 y estaba validado por las simulaciones. Sin embargo, este, que es ocupado para el proceso de selección universitaria, no ha sido posible de obtener para ser utilizado como instrumento.

${ }^{5}$ En el caso de Chile, hay carreras TP dictadas por universidades. Ello requiere entonces que estas IES opten e informen a los comités respectivos sobre su adscripción al sistema de acceso TP o universitario.
} 
quedado aceptados en caso de haberlo sido. A diferencia del subsector universitario, en esta fase no existirán listas de espera. Junto al aviso de selección, se informará el plazo en que los postulantes pueden hacer efectiva la matrícula. En dicho plazo, las instituciones adscritas al sistema de admisión podrán matricular únicamente a aquellos alumnos que hayan participado de la primera fase del proceso de postulación y los podrán matricular solo en la carrera donde fue aceptada su postulación.

Como resultado de la primera fase es previsible que haya desajustes importantes, como estudiantes que no quedaron en ninguna de sus opciones o que no estaban bien informados del proceso o que no hicieron efectiva su matrícula, dejando programas con vacantes disponibles. Para evitar los costos de ese desajuste, que en el sector universitario se resuelve con listas de espera y que para el sector TP se harían especialmente importantes en la transición, operará una segunda fase en la que se abrirá un nuevo período de matrícula por un plazo determinado, con características similares al existente en la actualidad; esto es, que los alumnos se matriculan por orden de llegada. Es decir, en esta segunda fase los estudiantes podrán matricularse directamente en aquellos programas donde aún queden vacantes disponibles (pues no se llenaron en la primera fase), utilizando los mecanismos definidos por la institución.

Es esperable que, en la medida en que el sistema se haga conocido, las vacantes llenadas en el primer proceso sean mayores. Asimismo, que las instituciones vayan simplificando el proceso de selección, por ejemplo, agrupando carreras similares que compartan un mismo plan común, bajo una misma denominación. Lo anterior haría que, progresivamente, la segunda fase fuera cada vez menos necesaria.

\section{Conclusiones}

En este artículo se han desarrollado argumentos que indican la naturaleza que debiera tener el sistema de acceso a la educación superior en el subsector TP requerido por ley en Chile. Se han remarcado algunas ventajas que podrían alcanzarse en el caso de pasar desde el sistema descentralizado vigente, a uno centralizado, especialmente en el contexto de una creciente presión por estudios superiores producto de las existentes ayudas financieras estatales.

El problema, motivado por el caso de Chile, existe también en la mayoría de los países de menor desarrollo, donde la cobertura escolar 
se ha acelerado y la formación en universidades debe evitar una masificación que la haga perder el nivel de su calidad. En ese contexto, en un sistema de educación superior donde la autoridad define estándares de calidad para las instituciones y financia a los estudiantes, resulta natural que el Estado defina el marco para financiar a los estudiantes, a quiénes priorizará y qué esquemas deben regir de modo de impedir la discriminación.

El diseño del mecanismo de acceso ha estado sujeto a una enorme controversia. Más allá de los instrumentos que puedan definirse, la mera existencia de un sistema de acceso centralizado, o coordinado, ha solido ser resistida por muchas instituciones. Sin embargo, el grado de centralización de un sistema de acceso no tiene que ver con lo selectivo que este sea. A menos que el sistema centralizado requiera a todas las instituciones usar instrumentos de alta selectividad y usar ponderaciones altas, el sistema puede convivir con instituciones de distinta selectividad; son ellas las que definirían cuán selectivas son, no el sistema.

Se han identificado, asimismo, los principales desafíos que conlleva implementar un sistema de admisión centralizado para el sector técnico-profesional de educación superior, partiendo por mantener los beneficios del descentralizado actual, que posee gran flexibilidad y cuyas instituciones son poco selectivas.

A partir del diagnóstico, se presentan aquí elementos para elaborar una propuesta de implementación de un sistema que, aunque más general, hemos aplicado al caso de Chile. La propuesta, como mostramos, cumple con reducir los costos de postulación y la ineficiencia vinculada al problema de unraveling. Además acepta diversos instrumentos que pueden ser propios de la tradición, así como objetivos específicos de las instituciones. Sin embargo, lo más importante es que minimiza el costo potencial asociado a la falta de información, a la multiplicidad de alternativas (muchas irrelevantes) y a las vacantes que no pudieran ser llenadas en una primera etapa del proceso de postulación.

Entre los mayores desafíos que permanecen se encuentra el hecho de que, si bien la descoordinación dentro del sistema TP se reduce, ella no se resuelve. En efecto, este sistema se hace obligatorio para un conjunto de instituciones, no para todas. Por ello, las exentas de tal obligación tendrán el incentivo de adelantar su proceso, lo cual afectaría adversamente a las que opten por este sistema, en particular si ellas son obligadas a pertenecer. Como contrapartida puede señalarse que tal 
situación estaría reducida a un subconjunto pequeño de la matrícula, lo que ocurre en el caso del SUA. Además, podría esgrimirse que la pertenencia al sistema de admisión ha sido buscada por las propias instituciones. De cualquier forma, tiene lógica explorar ya sea la obligatoriedad para todas las instituciones que reciben financiamiento público o, al menos, la limitación sobre las fechas en que pueden iniciar sus procesos de matrícula quienes no adscriben al sistema de acceso (por ejemplo, después de concluida la primera fase).

Por su parte, el sistema tampoco coordina con el sistema de acceso universitario. La sustitución reflejada en las preferencias de los estudiantes entre instituciones de un mismo subsistema es a veces menor que aquella entre instituciones de distintos subsistemas. En este sentido, la diferenciación administrativa entre sistema universitario y TP puede carecer de toda lógica, y debiera pensarse más en una conceptualización regional o de otra categoría que se haga más cargo de esa relación.

Un camino a evaluar - la integración completa entre los sistemas - no parece razonable, al menos en una etapa inicial, toda vez que la irrupción de un nuevo sistema podría dañar el funcionamiento del otro, que ya tiene un buen rodaje. Sin perjuicio de transitar en esa dirección y dado que el subsistema universitario tiene mayor tradición y menos incertidumbre, tiene más sentido que se haga coincidir la resolución del sistema universitario con la primera fase del subsector TP. Como únicamente el sistema TP tendría una segunda fase, que le otorga mayor flexibilidad, la resolución final de este sistema sería posterior.

Finalmente, debe considerarse la aplicabilidad de algunas de las ideas planteadas para el sistema de acceso TP al mundo universitario. Un problema mayor es que el SUA establece ponderaciones mínimas en algunos instrumentos, lo cual — según algunas instituciones - no tiene sentido, en especial para las menos selectivas. Algunas de ellas no solo están orientadas a un segmento de menor formación previa, sino que tienen métodos de enseñanza (o pudieran llegar a desarrollarlos) que les son propios. Así, una adscripción a un sistema obligatorio, con ponderaciones mínimas, las dejará fuera del sistema de acceso y con ello las restará de beneficios estudiantiles (como la gratuidad). La forma de resolver este problema es que, también para el mundo universitario, el sistema de acceso libere el número de instrumentos y sus ponderaciones. Ello, de paso, serviría directamente a un conjunto de universidades que imparten carreras técnicas. 


\section{Bibliografía}

Abdulkadiroglu, A., Nikhil, A. y Parag, P. 2017. The Welfare Effects of Coordinated Assignment: Evidence from the NYC HS Match. American Economic Review 107(12), 3635-3689.

Aequalis 2019. Subsistema de acceso a la formación técnico-profesional: reflexiones y propuestas desde la experiencia en formación para el trabajo. Disponible en: https://aequalis.cl/sites/default/files/publicaciones/2019-07/subsitema-deacceso-a-la-formacion-tecnico-profesional.pdf [25 de noviembre 2019].

Bernasconi, A. y Rojas, F. 2003. Informe sobre la educación superior en Chile: 1980-2003. Instituto de la UNESCO para la Educación Superior en América Latina y el Caribe (IESALC-UNESCO). Disponible en: http://www.iesalc. unesco.org.ve/dmdocuments/biblioteca/libros/14.pdf [23 de noviembre 2019].

Brunner, J. 2012. Ranking de notas: ¿equidad o confusión? Disponible en: http:// www.brunner.cl/?p=2752 [20 de marzo 2020].

Comisión de Financiamiento Estudiantil para la Educación Superior 2012. Análisis y recomendaciones para el sistema de financiamiento estudiantil. Ministerio de Educación, Chile. Disponible en: http://200.6.99.248/ bru487cl/files/ ComParedes.pdf [25 de noviembre 2019].

DEMRE 2010. Prueba de Selección Universitaria (PSU): antecedentes y especificaciones técnicas. Documento interno. Departamento de Evaluación, Medición y Registro Educacional. Santiago: Vicerrectoría de Asuntos Académicos, Universidad de Chile.

DEMRE 2016. Prueba de Selección Universitaria. Informe técnico. Vol. I: Características principales y composición. Disponible en: https://psu.demre. cl/estadisticas/documentos/informes/2016-vol-1-caracteristicas-principales-ycomposicion.pdf [22 de noviembre 2019].

DEMRE 2019. Proceso de admisión 2020. Disponible en: https://psu.demre.cl/ calendario/ [10 de octubre 2019].

Ekmekci, M. y Yenmez, M.B. 2014. Integrating Schools for Centralized Admissions. GSIA Working Papers N²014-E20. Carnegie Mellon University.

Espinoza, R., Soohyung, L. y López, H. 2017. Endogenous Market Formation: Theory and Evidence from Chilean College Admissions. Documento de Trabajo $\mathrm{N}^{\circ}$ 2017-002. Disponible en: https://www.waseda.jp/inst/wias/ assets/uploads/2017/08/002_Lee_Endogenous-Market-Formation.pdf [20 de noviembre 2019].

Gil, F.J., Paredes, R. y Sánchez, I. 2013. El ranking de notas: inclusión con excelencia. Temas de la Agenda Pública 8(60), 3-19.

Grassau, E. 1956. Análisis estadístico de las pruebas de Bachillerato. Anales de la Universidad de Chile 102, 77-93.

Halaburda, H. 2009. Unravelling in Two-Sided Matching Markets and Similarity of Preferences. Working Paper N 09-068. Harvard Business School. Disponible 
en: https:/www.hbs.edu/faculty/Publication\%20Files/09-068_13002cf0-a22d49c1-9359-b855e92f8818.pdf [20 de marzo 2019].

Koljatic, M. y Silva, M. 2006. Validación de la PSU: comentarios al estudio acerca de la validez predictiva de los factores de selección a las universidades del Consejo de Rectores. Estudios Públicos 104, 331-346.

Machado, C. y Szerman, C. 2016. Centralized Admission and the Student-College Match. Discussion Paper Series $N^{\circ}$ 10251. Institute of Labor Economics (IZA).

Mineduc 2018. Estrategia Nacional de Formación Técnico-Profesional. Santiago: Mineduc, Unesco.

Mineduc 2019. ¿Qué sabemos sobre admisión a la educación superior? Una revisión para la implementación del nuevo Sistema de Acceso en Chile. Santiago: Centro de Estudios Mineduc.

Niederle, M., Roth, A. y Ünver, M.U. 2009. Unraveling Results from Comparable Demand and Supply: An Experimental Investigation. Working Paper $\mathrm{N}^{\circ} 10-$ 095. Harvard Business School. Disponible en: https://hbswk.hbs.edu/item/ unraveling-in-two-sided-matching-markets-and-similarity-of-preferences [30 de noviembre 2019].

OECD 2018. Education at a Glance 2018: OECD Indicators. Paris: OECD Publishing. Disponible en: http://dx.doi.org/10.1787/eag-2018-e [27 de noviembre 2019].

Orr, D., Usher, A., Haj, C., Atherton, G. y Geanta, I. 2017. Study on the Impact of Admission Systems on Higher Education Outcomes. Vol. 1: Comparative Report. Luxembourg: European Commission, Publications Office of the European Union.

Paredes, R. 2018. Desafíos en educación superior técnico-profesional (413-444). En Sánchez, I. (ed.), Ideas en educación. Vol. II: Definiciones en tiempos de cambio. Santiago: Ediciones de la Pontificia Universidad Católica de Chile.

Paredes, R. y Sevilla, P. 2015. Reforma educación superior técnico-profesional (317-345). En Sánchez, I. (ed.), Ideas en educación. Reflexiones y propuestas desde la UC. Santiago: Ediciones de la Pontificia Universidad Católica de Chile.

Ruiz-Tagle, C. y Paredes, R. 2019. Educación superior técnico-profesional. ¿Una alternativa a la universitaria? El Trimestre Económico 86(341), 31-63.

Roth, A.E. 1984. The Evolution of the Labor Market for Medical Interns and Residents: A Case Study in Game Theory. Journal of Political Economy 92(6), 991-1016.

Roth, A.E. 1991. A Natural Experiment in the Organization of Entry-Level Labor Markets: Regional Markets for New Physicians and Surgeons in the United Kingdom. The American Economic Review 81(3), 415-440.

Sevilla, P., Farías, M., Weintraub, M. 2014. Articulación de la educación técnicoprofesional: una contribución para su comprensión y consideración desde la política pública. Calidad en la Educación 41, 83-117.

UDP-Vertebral 2019. Regímenes de admisión en la educación superior técnicoprofesional. Enfoque de Políticas ESTP N ${ }^{\circ} 1$. 


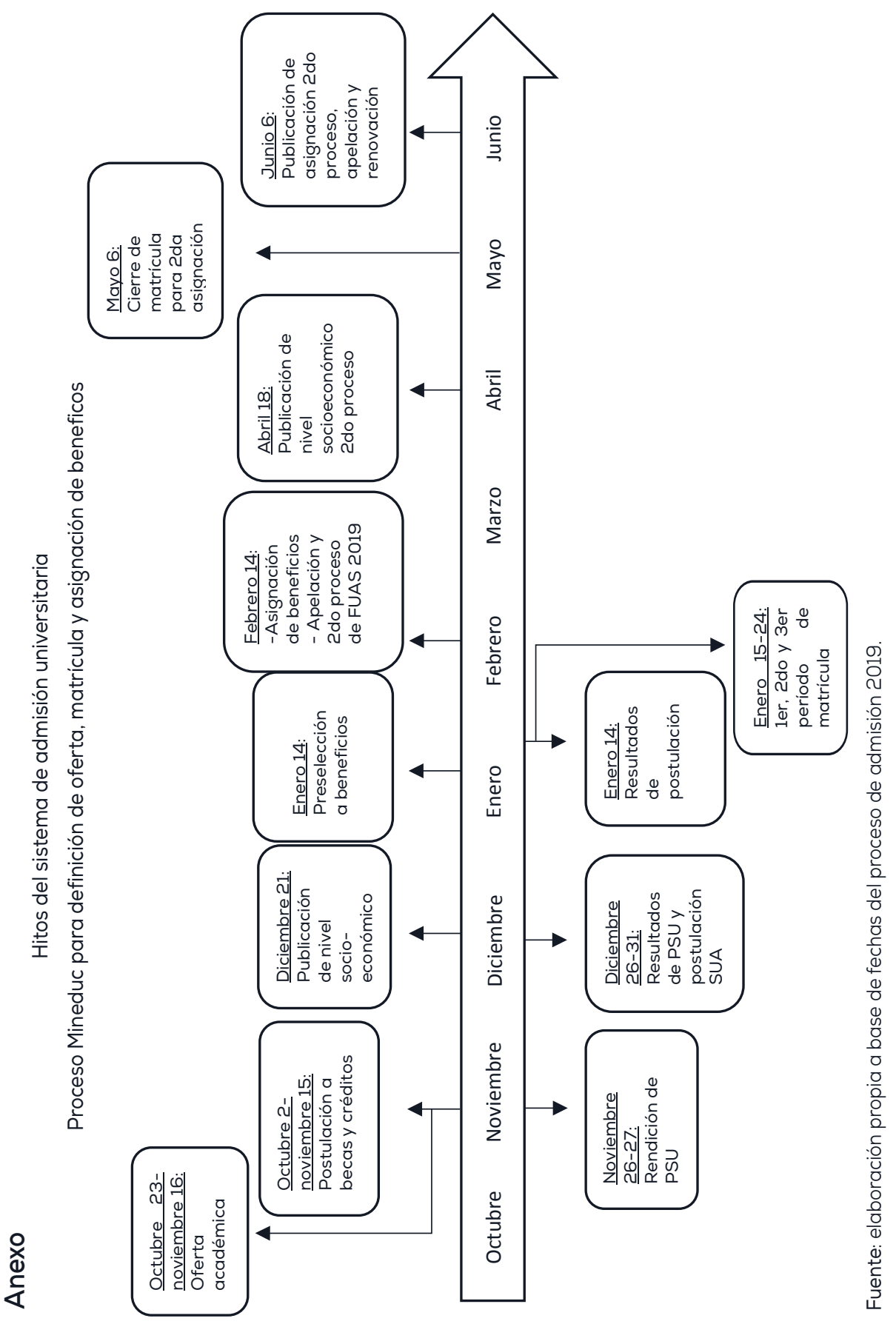

